\title{
Size Confinement Effects in AgX Nanocrystals as Studied by Cryo-EFTEM and EELS
}

\author{
V. P. Oleshko
}

Department of Materials Science \& Engineering, University of Virginia, Charlottesville, VA 22904

Considerable complexity in the valence-band structure caused by hybridization of halogen p-states and Ag 4d-states and occurrence of a low energy indirect gap lead to unique size restriction effects on electronic properties of nanocrystalline (NC) silver halides, when the particle size is below 100 $\mathrm{nm}$ [1]. In this case surface recombination of excitons becomes a dominant relaxation channel at the scale much larger than the Bohr radius of the indirect exciton $\sim 3 \mathrm{~nm}$. Due to an opportunity to probe excitations caused by inelastic electron scattering with high spatial $(0.1-1.0 \mathrm{~nm})$ and spectral $(0.1$ $1.5 \mathrm{eV}$ ) resolution, energy-filtering TEM and electron energy-loss spectroscopy (EFTEM/EELS) enable to make insights into unusual photophysical properties of nanocrystalline AgX matter in the size range where deviations from the bulk crystal selection rules occur. In this work, cryo-EFTEM and EELS have been employed to study size confinement effects of excitons in arrays of 3-5 particles and in individual $\mathrm{AgBr}_{0.95} \mathrm{I}_{0.05} \mathrm{NCs}$ of $22-185 \mathrm{~nm}$ in diameter.

Tuning the energy loss enables visualizing local excitations due to interband transitions (a low-loss fine structure at 4-18 eV), the bulk plasmon (22-23 eV) followed by a decrease in the background and intersection with inner-shell excitations (56-70 eV) [2,3]. Polarization waves formed by free and iodide-bound excitons of Mott-Wannier (MW) type are prominent excitations for $\operatorname{Ag}(\mathrm{Br}, \mathrm{I})$ $[1,2]$. Selection of the losses at $\mathrm{E}=16 \pm 5 \mathrm{eV}$ (Fig. 1) revealed in a weak rim around the NCs caused by polarization of exciton states by Coulomb field of striking electrons. Such rims can even extend at distances of a few nm away from the particle, which fit satisfactorily to the expected exciton size for $\mathrm{AgBr}$ [1]. No rims were observed in the zero-loss mode. Positioning the energy window at $\mathrm{E}=25 \pm 5 \mathrm{eV}$ enabled visualizing volume plasmons (VPs) in the NC. The non-uniform contrast and darker regions of $5 \mathrm{~nm} \times 10 \mathrm{~nm}$ in size are referred to predominant VP excitations confined within a $\theta$-shape volume inside the NC (Fig. 1, inset). When the fields due to surface (SP) losses reach throughout the structure, they couple and the probability for their generation becomes periodic in the size of the system. Both plasmons and excitons are considered to be coherent superposition of electron-hole excitations corresponding to transitions from occupied to unoccupied states. Since electronic sum rules must be satisfied, the surface excitations necessarily reduce the strength of the bulk excitations. As a result, the VP losses should exhibit similar periodic behavior. Coupling of SP and VP losses can induce periodic oscillations of their intensities with the particle size and induced charge (the intensity profile across the $\mathrm{NC}$ at $25 \pm 5 \mathrm{eV}$ in Fig. 1), thus demonstrating a size confinement of beam-induced excitations of valence $4 d\left(\mathrm{Ag}^{+}\right)$and $4 \mathrm{p}\left(\mathrm{Br}^{-}\right)$electrons due to contributions to the energy-level structure from carrier confinement and surface states. The decreased background at $\mathrm{E}=50 \pm 5 \mathrm{eV}$ leads to a contrast reversal that allows to observe tales of both SP and VP excitations with SPs localized within a 10-20 nm-thick surface layer.

Figs. $2 \mathrm{a}$ and $2 \mathrm{~b}$ present the Kramers-Kronig-derived complex refractive index $\mathrm{N}=\sqrt{\varepsilon}=\mathrm{n}+\mathrm{ik}$, and absorption coefficient, $\mu=2 \mathrm{Ek} / \mathrm{hc}$, for NCs with the mean equivalent diameter, $\mathrm{d}_{\mathrm{c}}$, of $50 \pm 4 \mathrm{~nm}(1)$ and $109 \pm 7 \mathrm{~nm}(2)$, a tabular AgBr microcrystal (t-MC) of $102 \pm 20 \mathrm{~nm}$ in thickness (3) and ab initio LMTO-ASA calculations for $\mathrm{AgBr}$ (4), respectively. For smaller NCs 1 (Fig. 2a), the imaginary 
part of refractive index (extinction coefficient), $\mathrm{k}$, shows an increased intensity of the band at $4 \mathrm{eV}$ (direct exciton transition at $\Gamma$ point, $\left(\Gamma_{8}^{-}, \Gamma_{6}^{-} \rightarrow \Gamma_{6}^{+}\right)$) relatively to the bands at $7 \mathrm{eV}$ and $10 \mathrm{eV}$ (unresolved $\left(\mathrm{X}_{6}^{-}, \mathrm{X}_{6}^{-}, \mathrm{X}_{7}^{-}\right) \rightarrow \mathrm{X}_{6}^{+}$) transitions at $\mathrm{X}$ point). The latter correlates with a size-confined enhancement of excitonic luminescence from $\mathrm{AgBr}$ NCs in the same size range [1]. The absorption coefficient exhibits size-dependent variations at $4 \mathrm{eV}$ (s- and p-states, the $1^{\text {st }}$ conduction band), at $\sim 7.5 \mathrm{eV}$ (mixed states, the $2^{\text {nd }}$ conduction band), at 12.2-13.6 eV and at 19-20.5 eV (p- and d-mixed states, the $3^{\text {rd }}$ conduction band), respectively, as compared to a AgBr t-MC and ab initio LMTOASA calculations for $\mathrm{AgBr}$ performed taking into account only direct transitions above $4.3 \mathrm{eV} \mathrm{[3].}$

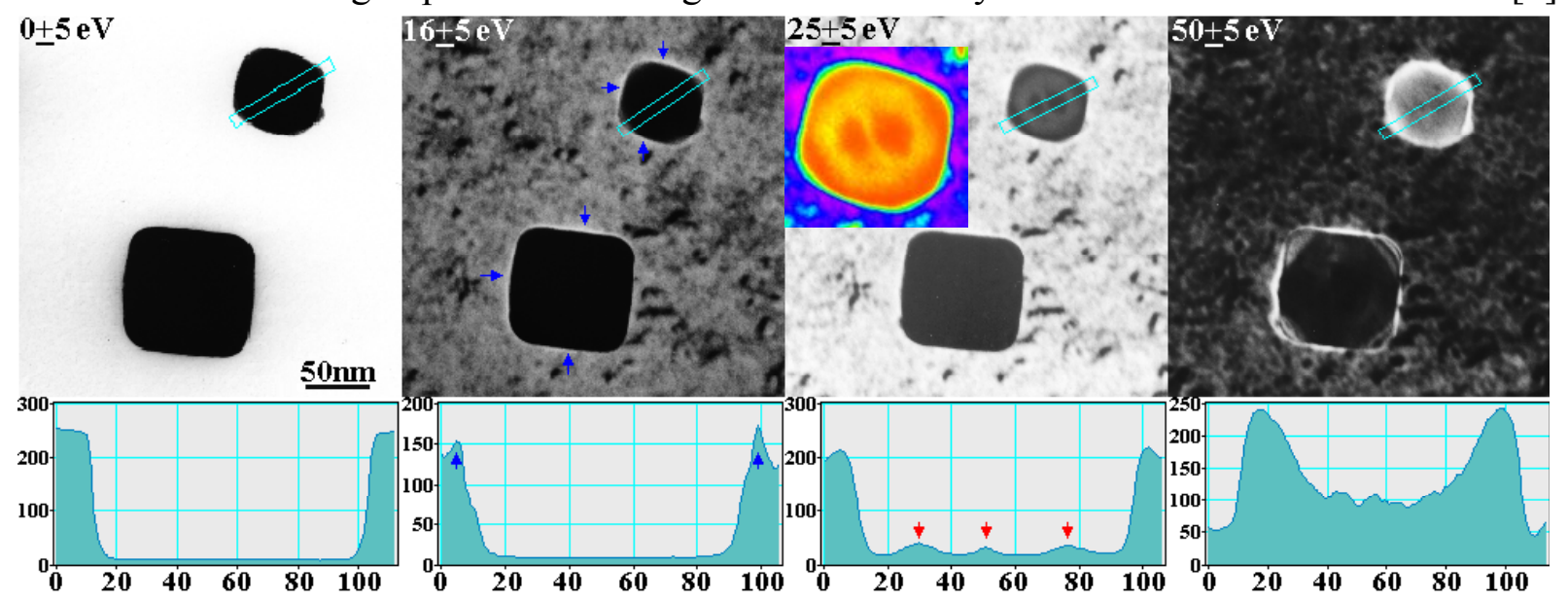

Fig. 1. Cryo-EFTEM of $\operatorname{Ag}(\mathrm{Br}, \mathrm{I}) \mathrm{NCs}(2)$ with a $10 \mathrm{eV}$ window at $0,16,25$ and $50 \mathrm{eV}$, respectively, and intensity profiles across the right upper $\mathrm{NC}$ as marked by rectangular boxes, a 30 pixel integration width. Up arrows point to MW exciton states at $16 \pm 5 \mathrm{eV}$, down arrows indicate periodical intensity oscillations caused by confined coupling VP and SP losses. An enlarged pseudocolor image of the NC (inset) shows a lateral distribution of VP losses.
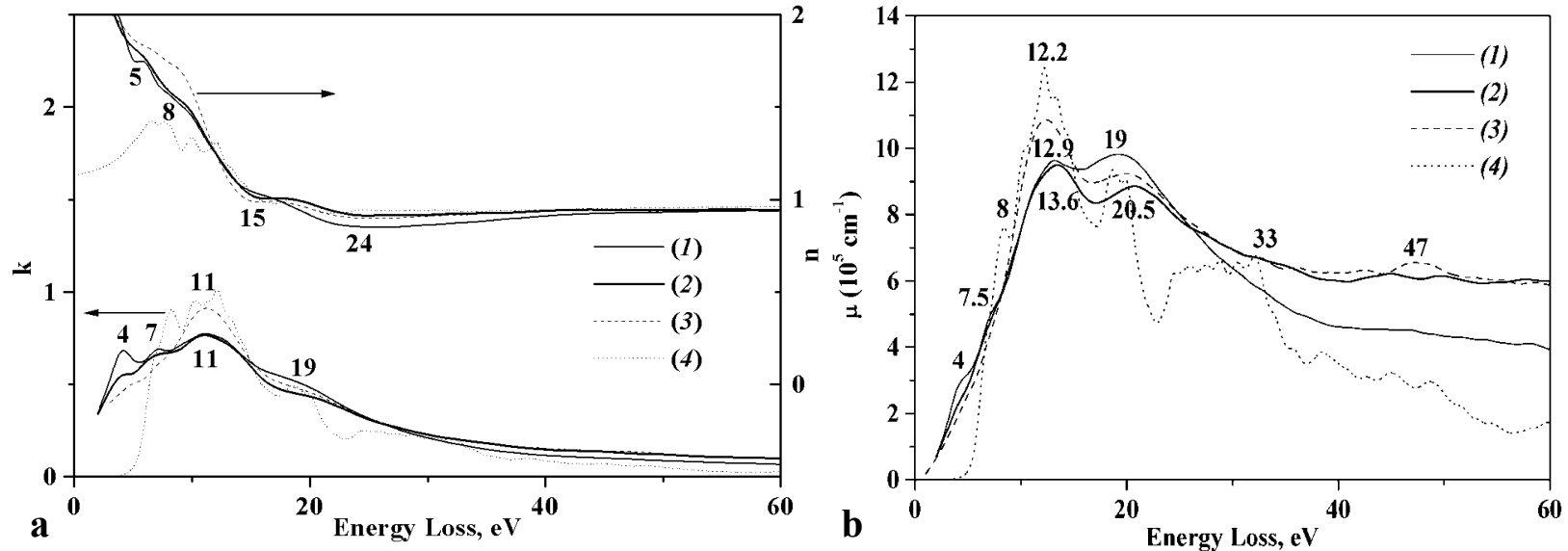

Fig. 2. (a) Complex refractive index, $\mathrm{N}=\sqrt{\varepsilon}=\mathrm{n}+\mathrm{ik}$, and (b) absorption coefficient, $\mu=2 \mathrm{Ek} / \mathrm{hc}$, for $\operatorname{Ag}(\mathrm{Br}, \mathrm{I}) \mathrm{NCs}$ with $\mathrm{d}_{\mathrm{c}}=50 \pm 4 \mathrm{~nm}(1)$ and $\mathrm{d}_{\mathrm{c}}=109 \pm 7 \mathrm{~nm}(2)$ in comparison with a $\mathrm{AgBr} \mathrm{t}-\mathrm{MC}$ of $102 \pm 20 \mathrm{~nm}$ in thickness (3) and ab initio LMTO-ASA calculations for $\mathrm{AgBr}$ (4). Curves fall to 0 at $\mathrm{E}<3 \mathrm{eV}$ taking into account the indirect exciton band gap, $\mathrm{E}_{\mathrm{g}}^{\mathrm{i}}$, at $2.68 \mathrm{eV}$.

References: [1] M. I. Freedhoff et al., J. Luminesc. 70 (1996), 400. [2] V. Oleshko et al., Nanostruct. Mater. 10 (1998), 1225; 11 (1999), 687. [3] V. Oleshko et al., Phys. Rev. B 67 (2003) 115409. 